\title{
Labyrinthe
}

21 | 2005 (2)

Communauté en pièces : d'Europe, d'Islam et d'ailleurs

\section{La communauté revendiquée}

Emplois politiques des termes du collectif en contextes

\section{Élise Massicard}

\section{(2) OpenEdition}

Journals

Édition électronique

URL : http://journals.openedition.org/labyrinthe/915

DOI : 10.4000/labyrinthe.915

ISSN : 1950-6031

Éditeur

Hermann

Édition imprimée

Date de publication : 22 juin 2005

Pagination : 103-109

Référence électronique

Élise Massicard, «La communauté revendiquée », Labyrinthe [En ligne], 21 | 2005 (2), mis en ligne le 16 juillet 2008, consulté le 22 avril 2019. URL : http://journals.openedition.org/labyrinthe/915; DOI :

$10.4000 /$ labyrinthe. 915

Propriété intellectuelle 


\section{LA COMMUNAUTÉ REVENDIQUÉE Emplois politiques des termes du collectif en contextes}

Élise MASSICARD

Bien que la qualification des alévis de «communauté» semble loin d'être évidente d'un point de vue scientifique, le chercheur ne peut faire l'impasse sur cette notion: même si la communauté alévie n'existe pas d'un point de vue étique, elle est objet de conceptualisations par les acteurs et existe donc de fait. La distinction systématique entre les usages politique et savant de la communauté semble un prémisse indispensable. L'analyse des représentations de la «communauté», de leurs termes ainsi que de leurs usages permet de comprendre les processus de construction identitaire, les termes dans lesquels ils se posent, ainsi que leurs enjeux.

L'emploi par des alévis du terme «communauté» pour qualifier le groupe a souvent plus le statut de revendication que de réalité sociologique ou même de perception. Le risque de travailler sur un groupe que certains de ses membres qualifient de communauté est celui de se réapproprier le discours des acteurs ${ }^{1}$ et d'occulter les dimensions proprement politiques de ce dernier ${ }^{2}$. De cette approche découle donc la nécessité d'établir une distinction claire entre le groupe (les alévis) et le mouvement qui s'en réclame et qui le construit en communauté (les alévistes).

Dans quelles circonstances la « communauté » alévie est-elle revendiquée, et comment? Nous passons ici de la question de la communauté à celle du communautarisme ${ }^{3}$. Mais toute revendication identitaire, de

1. Max Weber, Le Savant et le Politique, Paris, UGE, 1963.

2. Cette dimension est développée dans notre «Être pris dans le mouvement: savoir et engagement sur le terrain», Cultures et Conflits $\mathrm{n}^{\circ} 47$, décembre 2002, p. 117-143; disponible sur: http://www.conflits.org

3. Olivier Roy, Les Recompositions identitaires dans le monde musulman, Texte d'introduction pour le doctorat sur travaux, sous la direction de Rémy Leveau, Paris, Institut d'études politiques, 1995. 
reconnaissance au nom d'un groupe, peut-elle être ipso facto qualifié de « communautarisme»?

\begin{abstract}
Communautarisme
La notion de "communautarisme » fait référence à une tradition anglosaxonne: il désigne une situation dans laquelle des «communautés sont des composantes reconnues, plus ou moins bien intégrées, d'ensembles plus vastes, souvent nationaux. Alors que le particularisme consiste souvent à revendiquer la reconnaissance d'une spécificité par l'État, le communautarisme implique que cette étape est déjà réalisée et que les revendications portent sur davantage de concessions, de droits collectifs ${ }^{4}$. De ce fait, le communautarisme est honni dans les contextes prônant la supériorité de l'individu sur le groupe et refusant l'idée de droits collectifs, par exemple en France. On voit ici que la qualification même de ces mouvements est liée également aux contextes sociaux et politiques.
\end{abstract}

La mobilisation aléviste vise à construire le groupe et à susciter la communauté, fût-elle imaginée, donc le sentiment d'appartenance. Ainsi les sites internet alévistes donnent-ils l'impression de l'existence d'une communauté alévie transfrontalière et unifiée 5 . D'où l'importance de symboles et de figures devant susciter l'identification produits, reproduits et brandis par les cadres du mouvement. Cependant, il semble ici aussi discutable de qualifier ce mouvement de communautariste au sens plein du terme.

Or, l'idéologie nationale unitariste de Turquie honnit à la fois la communauté et le communautarisme. On note la prégnance dans le discours politique des termes de l'unité, tels birlik (unité) bütünlük (totalité), birlik ve beraberlik (unité et concorde), mais aussi du spectre de la division, avec des termes tels bölücülük (séparatisme) ayırmak (séparer), et la conspiration comme tahrik et provokasyon (provocation), oyun (machination, complot), körüklemek (attiser), tezgahlamak (tramer), kışklrtmak (exciter, provoquer) ${ }^{6}$. Le traumatisme engendré par les velléités d'indépendance de différents groupes par rapport à l'Empire ottoman affaibli, encouragées par des puissances extérieures,

\footnotetext{
4. Christophe Jaffrelot, «Introduction: L'État face aux communautés», dans Cultures et Conflits, $\mathrm{n}^{\circ}$ 15-16, «État et communautarisme», automne-hiver 1994, p. 3-6.

5. Martin Sökefeld, «Alevism Online...», art. cit.

6. Voir pour les manuels scolaires Étienne Copeaux, Espaces et Temps de la nation turque. Analyse d'une historiographie nationaliste. 1931-1993, Paris, CNRS, 1997.
} 


\section{La communauté revendiquée}

plane encore; on le nomme couramment le «syndrome de Sèvres », ce traité de 1920 marquant le dépeçage de l'Empire et son occupation par les puissances occidentales, avant d'être annulé par le traité de Lausanne, suite à la guerre de libération menée par Mustafa Kemal. Dans ce cadre, toute velléité d'affirmer une spécificité est considérée comme potentiellement dangereuse.

Cette illégitimité de tout ce qui frise le particularisme et le communautarisme a des traductions directement juridiques, notamment dans l'impossibilité légale pour tout groupe revendiquant une spécificité de s'organiser (ce qui contraste nettement avec la situation en Grèce analysée par Jeanne Hersant dans ce numéro). Ainsi, l'article 5 de la loi de 1983 sur les associations interdit aux mezhep de s'organiser sous la forme d'associations ${ }^{7}$. En outre, selon le code civil, une fondation $\left(v a k l f^{8}\right)$ établie avec le but de soutenir un courant politique, une race ou une cemaat particuliers ${ }^{9}$ ne doit pas être enregistrée. Les groupes, et notamment les groupes religieux, ne peuvent donc s'organiser formellement que s'ils taisent leurs objectifs liés à la confession. De ce fait, la plupart des organisations évitent de mentionner, dans leurs statuts ou dans leur appellation officielle, le terme «alévi», mais exprimeront cette dimension par des périphrases ou de simples allusions et par des figures devenues symboles de l'alévité. Ainsi, même les cadres alévistes, qui s'efforcent pourtant de construire un groupe uni et d'obtenir une reconnaissance, ne revendiquent pas forcément de «communauté», notamment dans la mesure où celle-ci est souvent illégitime ${ }^{10}$, même si cela dépend des termes employés.

En Turquie, le seul statut juridique «communautaire» existant est celui de cemaat (qu'on pourrait traduire par «groupe religieux», et qui est souvent rendu par «communauté religieuse»). Ont été constitués en cemaat sous la République certaines anciennes millet ottomanes. $\leadsto$ «La communauté d'historicité...» « et $\leadsto$ «Réflexion sur les catégories du

\footnotetext{
7. Art. 5, § 2, 5 et 6, Dernekler kanunu, loi n 29008, 6 octobre 1983.

8. Bien de mainmorte, consistant en une somme en numéraire ou un bien foncier qu'un donateur offre ou lègue à une institution religieuse ou charitable. Pour une analyse détaillée du statut juridique des vaklf en Turquie contemporaine ainsi que de leur rôle dans la vie politique, on peu se référer à Faruk Bilici, «Sociabilité et expression politique islamistes en Turquie: les nouveaux vakıfs », Revue française de science politique, 43 (3), juin 1993, p. 412-434.

9. Article 101 du code civil.

10. Voir notre «Les alévis et le discours de l'unité en Turquie depuis les années 1980 », dans HansLukas Kieser (dir.), Aspects of Political Language in Turkey (19 $9^{\text {th }}-20^{\text {th }}$ Centuries), Istanbul, Isis, 2002.
} 
droit international...» ${ }^{-}$Ces groupes religieux non musulmans furent institutionnalisés au cours du XIX ${ }^{\mathrm{e}}$ siècle, souvent sous protection étrangère (française pour la millet catholique, anglaise pour la millet protestante, grecque pour la millet orthodoxe). Ce sont ces groupes non musulmans restant présents après les bouleversements de populations consécutifs à la Première Guerre mondiale et à la fondation de la République et reconnus par le traité de Lausanne (Juifs, Grecs, Arméniens) qui y ont acquis le statut de cemaat. Les alévis, qui n'avaient pas été institutionnalisés comme millet sous l'Empire ottoman, et n'ont pas été l'objet de protection internationale, n'ont pas été concernés par ce statut. Ils ne le revendiquent pas non plus, dans la mesure où c'est un statut «minorisant», c'est-à-dire marqué par une relation de domination non seulement numérique, mais aussi politique. Bien que jouissant des mêmes droits que les autres citoyens de Turquie et protégés par des accords internationaux, les membres des cemaat sont considérés comme des citoyens un peu à part, ne formant pas le cour de la nation ${ }^{11}$. En raison du «syndrome de Sèvres» et de leurs liens réels ou supposés avec des puissances étrangères dont les intérêts ne sont pas toujours ceux de la Turquie, leur allégeance et leur loyauté sont souvent considérées comme douteuses.

Si l'expression «alevi cemaati» (communauté alévie) est parfois employée et revendiquée ${ }^{12}$, c'est moins en référence à ce statut que pour affirmer le caractère unifié du groupe, souvent quand on parle d'un patrimoine. Cela fait en outre plutôt référence à une autre acception du terme. En turc contemporain en effet, celui-ci désigne également de nouveaux groupes religieux islamiques apparus durant la seconde moitié du $\mathrm{XX}^{\mathrm{e}}$ siècle. Ils se différencient des confréries classiques (tarikat) en ce qu'ils entretiennent rarement une pratique mystique, et sont moins hiérarchisés. Divisées en un grand nombre de branches, les cemaat dominent aujourd'hui la mouvance religieuse turque (Nurcu, Süleymanc1). Actifs notamment dans le domaine de l'éducation, ils sont aussi à l'origine de la construction de mosquées et de l'entretien d'imams.

Ainsi, en Turquie, le terme «cemaat» ne correspond-il qu'imparfaitement aux représentations des acteurs eux-mêmes, y compris des

11. Voir notamment les travaux de Rifat Bali au sujet des Juifs de Turquie.

12. Voir par exemple «"Tayyıp Erdoğan bölücü ve halk düşmanı"», Cumhuriyet, 19 septembre 1994. 
entrepreneurs identitaires; ils l'emploient moins que d'autres termes. Est par exemple utilisé, dans le registre religieux, le mezhep (voir supra). Ce terme a l'avantage de faire un parallèle avec l'école de droit chaféite, dont l'existence est reconnue en Turquie, et donc d'articuler une spécificité religieuse qui reste légitime; cependant, il ne correspond à aucun statut juridique précis, et ne porte aucun bénéfice concret. Certains, qui tentent d'affirmer la nature musulmane de l'alévité, la qualifient également de tarikat, ce qui renvoie à une dimension confrérique et mystique. Cependant, ce statut est également peu revendiqué, notamment parce qu'il est illégal - les confréries sont interdites depuis 1925, ce qui n'empêche pas certaines de vivre dans une semi-clandestinité - et que ce terme a souvent une connotation de traditionalisme arriéré. C'est ainsi que ce terme est souvent employé par les détracteurs des alévis, voulant ainsi signifier l'illégitimité de ce phénomène. Nous avons, lors d'un épisode précis, tenté d'analyser de plus près les enjeux des différentes conceptualisations par les acteurs pour revendiquer, légitimer ou au contraire délégitimer le collectif alévi ${ }^{13}$.

Mais ces termes sont moins employés que ceux de Alevi toplumu ou topluluğu, ou tout simplement Aleviler (les alévis). Ces deux termes, que l'on pourrait aussi traduire par communauté, collectivité ou société alévie n'ont pas de connotation religieuse, à la différence des termes précédemment évoqués. Au contraire, ils évoquent le discours socialiste ou de gauche, et ont une connotation laïque, voire gauchisante ${ }^{14}$.

La fluctuation des termes du collectif invoqués pour construire et affirmer le groupe alévi s'explique tout d'abord par le fait qu'il n'existe pas d'équivalent pour l'alévité de l' "oumma» musulmane (la communauté des croyants). En outre, ces différents termes renvoient pour certains et dans certaines circonstances à des statuts juridiques définis, mais aussi à des connotations légitimes ou illégitimes. Cette fluctuation s'explique enfin par la diversité des représentations en jeu et des objectifs visés par les cadres associatifs alévistes. Certains visent en effet à la reconnaissance d'une spécificité religieuse dans le cadre de l'islam, d'autres à la simple absence de discrimination, ou à la reconnaissance de spécificités pas nécessairement religieuses, mais

13. Élise Massicard, «Les alévis et le discours de l'unité...», art. cit.

14. «Biz ilerici ve laik, Atatürkçü bir topluyuz». [Nous sommes une collectivité progressiste, laïque, et kémaliste]. Cumhuriyet, 8 septembre 1994. 
aussi culturelles. En Turquie, d'une manière générale, les cadres alévistes se trouvent dans la situation paradoxale de vouloir obtenir une reconnaissance collective sans la minorisation qu'elle induit presque automatiquement dans ce contexte peu propice à la communauté. Ainsi, tous refusent unanimement la qualification de «minorité» qui leur est accolée par certains rapports, notamment européens ${ }^{15}$; s'ils désirent voir leur spécificité reconnue, ils refusent catégoriquement d'être considérés comme des citoyens de seconde zone, mais veulent être reconnus en même temps comme étant au cœur de la nation. La différence patente avec la configuration en Grèce, analysée dans ce numéro par Jeanne Hersant, montre à quel point l'existence de statuts influence aussi les formes de revendication.

Il est cependant intéressant de remarquer que c'est en migration que les cadres alévistes utilisent le plus les termes équivalents à notre «communauté». En France tout d'abord, où mes quelques interlocuteurs alévistes ont tous employé spontanément le terme de «communauté alévie ${ }^{16}$ »; en Allemagne également, où le terme «Religionsgemeinschaft» ( " communauté religieuse») correspond à un statut et à des attributions, que des organisations alévistes ont obtenu dans certains Länder depuis $2000^{17}$.

Une conceptualisation du mouvement aléviste dans son ensemble comme «communautariste» semble donc peu pertinente, d'abord parce qu'elle irait à l'encontre du contexte social et politique turc, ensuite parce qu'elle ne semble pas rendre justice aux revendications et représentations des acteurs, ni aux contraintes auxquelles ils sont confron-

15. Commission des Communautés européennes, Rapport régulier 2004 sur les progrès réalisés par la Turquie sur la voie de l'adhésion, Bruxelles, 6 octobre 2004, disponible sur:

http://europa.eu.int/comm/enlargement/report 2004/pdf/rr tr 2004 fr.pdf

16. Directeur de la Fédération des unions alévies de France, Paris, 12 octobre 1999; une femme très active dans les organisations alévistes, Paris, 6 et 26 octobre 1999.

17. Le terme «Gemeinde » (équivalent de notre «paroisse»), également à connotation religieuse, est aussi très employé, mais il se réfère au groupe de pratique cultuelle, ou encore au groupe localisé qu'une organisation alévie est susceptible d'interpeller, conformément à l'usage du terme « cemaat » par les alévis de Turquie que nous avons décrit plus haut. On parle alors de la «Gemeinde berlinoise». Un cadre de la Fédération des unions alévies d'Allemagne, Cologne, 16 décembre 2001; le directeur de la Fédération des unions alévies d'Allemagne, lors de l'inauguration d'un nouveau local et lieu de culte associatif en présence du ministre de l'Intérieur allemand, Berlin, 6 novembre 1999. Ce terme semble de plus en plus utilisé depuis la reconnaissance religieuse de certaines organisations alévistes. Ainsi, la Fédération des unions alévies en Allemagne a récemment changé la traduction de son acronyme en allemand en fédération des Gemeinden alévies en Allemagne. 
tés, ou alors seulement dans certains contextes et situations. Les termes de l'affirmation du groupe, voire peut-être ses représentations, nous semblent donc devoir être replacés dans leurs contextes sociaux et politiques de revendication et d'énonciation.

Mais ce mouvement identitaire entraîne-t-il une «communautarisation»? On a observé ces dernières années l'apparition, notamment dans les grandes villes, de lieux marqués comme alévis dans lesquels se rendent des personnes qui auparavant ne se connaissaient pas. Probablement ce mouvement a-t-il contribué au renforcement d'identification de certains alévis comme alévis, même si ce processus est difficile à mesurer. Quant à la question de savoir si, d'un point de vue sociologique - étique - les relations à l'intérieur du groupe alévi se sont « communautarisées »-par exemple, si l'on peut observer un regain de l'endogamie infra-alévie -, elle demanderait des données qui à ma connaissance n'existent pas. 